Luque, A. \& Carrión, J. (2013). El maestro de audición y lenguaje: formación, experiencia previa y acceso. Revista Electrónica Interuniversitaria de Formación del Profesorado, 16 (2), 39-54.

DOI: http://dx.doi.org/10.6018/reifop.16.2.181141

\title{
El maestro de audición y lenguaje: formación, experiencia previa y acceso
}

Antonio Luque de la Rosa, José Juan Carrión Martínez

Universidad de Almería

\section{Resumen}

Habiendo transcurrido veinte años de andadura y desarrollo profesional desde el surgimiento del maestro de audición y lenguaje ( $A L)$ itinerante, parece conveniente reflexionar sobre el papel que ha venido desempeñando esta figura con objeto de aprehender las consideraciones oportunas que mejoren los planteamientos a adoptar de cara a promover la calidad de su actuación educativa.

Por otra parte, si bien a nivel formativo el plan de estudio de estos profesionales se ha visto afectado y mermado en su especificidad a causa de la nueva estructura de grados y menciones, a nivel de ocupación laboral dicha figura se ha consolidado como especialidad docente a partir de la publicación del Real Decreto 1594/2011, de 4 de noviembre (BOE 9-112011).

Con este estudio se pretende contribuir a la comprensión de la construcción de este perfil profesional, realizando un recorrido por la formación que han recibido, la experiencia previa de carácter laboral que mantienen y la forma de acceso a los puestos de trabajo que desarrollan.

\section{Palabras clave}

Maestro de audición y lenguaje; formación inicial; formación permanente; experiencia laboral. 


\title{
The teacher of audition and language: education, experience and access
}

\begin{abstract}
Having spent twenty years of business and professional development from the emergence of speech and hearing teacher $(\mathrm{AL})$ traveling, it seems appropriate to reflect on the role he has been playing this figure in order to apprehend the things considered to improve the approach to adopt towards to promote the quality of its educational activities.
\end{abstract}

On the other hand, while at the training curriculum of these professionals has been affected and diminished in its specificity because of the new grading structure and terms, in terms of occupation that figure has become a teaching specialty from the publication of Royal Decree 1594/2011 of 4 November (BOE 11/09/2011).

This study aims to contribute to the understanding of the construction of this professional profile, making a tour of the training received, previous experience of maintaining a labor and how to access the jobs they perform.

\section{Key words}

Teacher of audition and language; initial training; lifelong learning; work experience.

\section{Introducción}

Si bien han venido existiendo en nuestro país perfiles profesionales dedicados a la atención específica de problemáticas relacionadas con la pronunciación y el habla (logopedas), surge en España al inicio de la década de los noventa una nueva figura docente, el maestro de audición y lenguaje, en cuya propia denominación se aprecia el deseo de cambio en dicho rol, promoviendo la superación de los planteamientos recuperadores y rehabilitadores de carácter terapéutico que afectan a determinados canales de comunicación (audición/fonación), para avanzar hacia una concepción de dicho profesional como potenciador del desarrollo comunicativo, cognitivo y sociopersonal a través del lenguaje en el conjunto del alumnado.

Si queremos analizar la acción educativa de los profesionales objeto de este estudio deberemos comenzar vislumbrando las variables experienciales que traen consigo al escenario educativo, entendidas como sus experiencias, modos de comprensión específicos y presupuestos que determinan su conocimiento y actuación (Tikunoff, 1979).

Así, si nos centramos en el análisis del rol educativo que viene desempeñando un docente, hemos de considerar que dicho perfil suele estar determinado por una serie de factores incidentes en la construcción de su conciencia profesional (Luque de la Rosa, 2008).

En este sentido, la primera faceta que consideraremos a la hora de analizar el pensamiento profesional por parte del maestro de audición y lenguaje $(A L)$ itinerante será la relacionada con el desarrollo de actuaciones profesionales previas que han experimentado antes de su incorporación a estos puestos de trabajo.

Además de la experiencia previa, en la construcción de la autoconcepción profesional de estos docentes influirán las inconsistencias que éstos tienen entre su enseñanza práctica y la formación recibida, siendo necesario potenciar un proceso de reflexión entre los mismos 
que asegure el desarrollo profesional, la mejora del conocimiento y la efectiva creación, asentamiento y reformulación de las propias creencias profesionales (Clarke y Hollingsworth, 2002).

Según Pérez Gómez (1995) la práctica pedagógica ha tenido un proceso de madurez tanto en sus concepciones teóricas como en las proyecciones prácticas que le sirven de fundamento el cual ha incidido en la concepción de la adecuada formación del docente. En la actualidad "dicha formación debe desarrollarse a partir de las funciones, individualidades e intereses de los diversos colectivos docentes, proyectando cada uno su propia práctica a partir de un proceso de autorreflexión crítica, planeación y accionar estratégico" (Pérez Gómez, 1995, p.340).

Asumiendo que a lo largo de dicho desarrollo, -que abarca tanto el proceso de formación de la etapa inicial como permanente-, se construyen y reconstruyen en forma íntegra el conjunto de competencias profesionales del docente a través de los procesos culturales vividos por éste, -ya sea de modo personal o colectivo-, se concluye la importancia del ámbito formativo global en la construcción del pensamiento profesional del maestro de $\mathrm{AL}$ itinerante.

\section{Formación Inicial}

Según Gimeno Sacristán, Pérez Gómez, Martínez Rodríguez, Torres Santomé, Angulo Rasco y Álvarez Méndez (2009), la formación inicial que en la actualidad recibe el profesorado resulta insuficiente. Los cambios sociales operados en las últimas décadas -los cuales han conducido hacia una sociedad globalizada, con acceso a numerosas y diversas fuentes de información y con constantes movimientos migratorios en las poblaciones-, han dado lugar a unos nuevos contextos educativos a los que el profesorado debe hacer frente. Por lo cual, en este nuevo escenario, la formación que reciben los futuros docentes no suele responder a las necesidades profesionales que presenta su labor en el aula (Nuño Pérez, 1991).

\section{Formación Permanente}

Desde la implantación de la LOGSE se reafirmó la idea sobre la importancia que tiene la formación permanente en la labor que viene desarrollando el docente en las aulas. Así, en palabras de Escudero Muñoz (1993), "si bien la calidad de un sistema educativo pasa, sin duda, por muchos frentes, entre ellos, la formación continuada de los profesores ocupa uno de los lugares preferentes y prioritarios" (p.15). Reconstruir la profesión docente, dignificarla en sus diversas facetas, garantizar experiencias y oportunidades valiosas que hagan posible el desarrollo de nuevas concepciones, sentidos, propósitos y recursos metodológicos por el profesorado, representan elementos importantes para hacer posible la mejora de la educación. Pero, como expone Fullan (1993), "una formación continua del profesorado que posibilitara dichos cambios resulta ser también uno de los peores y más difíciles problemas de resolver" (p.14).

Por consiguiente, a nivel teórico llevamos más de dos décadas apostando por una concepción de la formación permanente como el periodo formativo que se inicia una vez concluido la formación inicial, el cual, siguiendo a Imbernóm Muñoz (2005), "más allá de recopilar datos o informaciones complementarias a la obtenida en el periodo inicial, ha de generar modalidades que ayuden al profesorado a descubrir su propia teoría, ordenarla, fundamentarla, revisarla y destruirla o construirla de nuevo" (p.73).

Esta concepción de la formación permanente del profesorado engarza con el planteamiento de pensamiento práctico de Schön (1983) al considerar de vital importancia para la misma el desarrollo de una reflexión sobre la acción y sobre la reflexión en la acción (reflexión crítica según Habermas (1987). Similar planteamiento expone Pérez Gómez al 
señalar que "la reflexión sobre la acción es un componente esencial del proceso de aprendizaje permanente que constituye la formación profesional” (Pérez Gómez, 2002, p.419).

En este sentido la formación permanente podría ser definida como un proceso recurrente que apela al protagonismo del docente en la continua ampliación de la visión de mundo (interno y externo) inherente a su labor profesional, incidiendo directamente en la construcción y reconstrucción de su pensamiento profesional.

Con relación a esta concepción de formación permanente, son diversos los autores que advierten de la conveniencia de establecer reformas en el sistema que se encarga de su desarrollo en la actualidad, afirmando Gimeno Sacristán y otros (2009) que "es necesario un cambio profundo en la metodología docente, en la orientación educativa, en la formación inicial y permanente del profesorado, en los currículos educativos y en los espacios y tiempos escolares" (p.52)

Habría que intentar que a lo largo del proceso de formación no se perdiera de vista el concepto de profesionalidad y el referente ideológico que subyace a esos discursos y procesos formativos (Fernández Sierra y Barquín Ruiz, 1998), porque en el momento actual, si no promovemos una formación profesionalizante y crítica en el docente e intentamos ligar nuestras acciones a sus fundamentos, es fácil que éstas se nos escapen arrastradas por las corrientes al uso, cada día más segregadoras y tecnificantes (Angulo Rasco, 1999).

\section{Objetivos}

El objeto-problema de este estudio consistirá en realizar un análisis de la situación que viven los profesionales itinerantes de audición y lenguaje, profundizando en el estudio de la formación recibida, su experiencia previa y la forma de acceso al puesto que desarrollan.

1. Apreciar las características que configuran y condicionan la actuación que realizan estos profesionales con su alumnado así como la concepción educativa que subyace a la misma.

2. Evidenciar el modelo de formación inicial y permanente que mantienen y las repercusiones que conlleva en la delimitación de su perfil profesional.

3. Conocer la experiencia previa que han desarrollado estos profesionales antes de su incorporación como maestros de audición y lenguaje y las implicaciones en su actuación docente.

4. Analizar, igualmente, si dicha actuación viene condicionada por el sistema de acceso al puesto que desempeñan y las características del mismo.

En síntesis, deseamos indagar la hipótesis de partida con relación a la permanencia en tal figura de un modelo de intervención clínico-terapéutico de problemas logopédicos, así como los factores que lo condicionan.

\section{Metodología}

\section{Participantes}

La investigación que ahora se presenta se incardina en un enfoque o paradigma interpretativo, y ello por la naturaleza del hecho investigado, por los propios fines de la actividad investigadora, por el modo de abordar el análisis e interpretación de los datos y por la metodología cualitativa de la que nos servimos para la toma de información. 
Consideramos que sólo desde esta perspectiva podemos acercarnos al conocimiento de esta realidad educativa (Goetz y LeCompte, 1988; Rodríguez Gómez, Gil Flores y García Jiménez, 1996; Taylor y Bogdan, 1986) puesto que la misma es cambiante y, el contexto social y los individuos que la conforman, la influyen y determinan.

Stake (1995) define el objetivo de la investigación cualitativa como la comprensión de las complejas interrelaciones que se dan en la realidad, centrando la indagación en los hechos.

\section{Participantes}

En la investigación que presentamos, la población hace referencia al conjunto de maestros itinerantes de audición y lenguaje, figura profesional que puede considerarse de un perfil muy exclusivo de España, con relación a la cual intentaremos analizar y comprender su actuación profesional, acercándonos a la práctica educativa que realizan, viviéndola y percibiéndola en su singularidad, con objeto de situar significativamente el conjunto de dimensiones que conforman esa realidad natural. Se ha tomado una selección de informantes formada a partir de un conjunto de 9 maestros especialistas en audición y lenguaje, que constituyen en el momento de la investigación la totalidad de los AL adscritos a la plantilla de Equipos de Orientación Educativa (en adelante, EOE) de Almería (España). Ver tablas 1 y 2.

Tabla 1. Número de sesiones de contacto con cada maestro de audición y lenguaje

\begin{tabular}{llllllllll}
\hline $\mathrm{AL}$ & 1 & 2 & 3 & 4 & 5 & 6 & 7 & 8 & 9 \\
\hline $\mathrm{N}^{\circ}$ contactos & 6 & 5 & 5 & 5 & 5 & 5 & 6 & 4 & 5 \\
\hline
\end{tabular}

Tabla 2. Siglas identificativas de cada tipo de informante

\begin{tabular}{|c|c|c|c|c|c|c|c|}
\hline$A L$ & $\mathrm{CO}$ & DD & & $\mathrm{Ma}$ & Or & PT & $\mathrm{Tu}$ \\
\hline $\begin{array}{l}\text { Maestro de } \\
\text { audición y } \\
\text { lenguaje }\end{array}$ & $\begin{array}{l}\text { Coordinador } \\
\text { de Equipo de } \\
\text { orientación } \\
\text { educativa }\end{array}$ & $\begin{array}{l}\text { Miembro } \\
\text { equipo } \\
\text { directivo }\end{array}$ & & Madre & Orientador & $\begin{array}{l}\text { Maestro de } \\
\text { pedagogía } \\
\text { terapéutica }\end{array}$ & $\begin{array}{l}\text { Maestro } \\
\text { tutor }\end{array}$ \\
\hline
\end{tabular}

\section{Diseño y análisis de datos}

El análisis e interpretación de los datos cualitativos de los que nos servimos para la toma de información (Goetz y LeCompte, 1988; Rodríguez-Gómez, Gil-Flores y García-Jiménez, 1996; Stake, 2007; Taylor y Bogdan, 1986; Woods, 1989), en una aproximación etnográfica de caso múltiple se ha desarrollado en varias etapas:

a) Acopio y transcripción de la información.

b) Reducción y depuración de información desde el criterio marcado por los interrogantes y objetivos.

c) Precategorización y construcción texto interpretativo.

d) Simplificación textual y de precategorías.

e) Categorización e informe final.

En el tratamiento de la información no se ha considerado pertinente el tratamiento a través de programas informáticos, pues se trataba de información siempre tomada en contexto de inmersión, tanto la de la entrevista como la de la observación, que requería un análisis interpretativo con un fuerte peso de la capacidad del propio investigador para su mejor 
aprovechamiento. De hecho, el mapa de categorías generado, no parte de un análisis precategorial, sino de la interpretación constructiva de la información y del conjunto de elementos didácticos y lingüísticos que el propio estado de la cuestión nos alumbraba (ver Tabla 3):

Tabla 3. Categorías

\begin{tabular}{ll}
\hline CATEGORÍAS & \\
\hline Didácticas & Lingüísticas \\
\hline Metodología & Componente fonético-fonológico \\
\hline Actividades & Componente semántico \\
\hline Implicación tutorial & Componente morfosintáctico \\
\hline Organización temporal & Componente pragmático \\
\hline Organización espacial & \\
\hline Recursos materiales & \\
\hline
\end{tabular}

\section{Instrumentos}

Se han utilizado instrumentos propios de la metodología cualitativa: entrevista en profundidad (en los resultados se codificará con E), observación de campo (en los resultados se codificará con $\mathrm{CC}$ ) y análisis de documentos (en los resultados se codificará con $A D)$ :

1) La entrevista en profundidad se ha configurado con un planteamiento abierto, sin un cuestionario-guía como tal, ya que las entrevistas se han contextualizado en una inmersión en los centros, tomándose como referencia de guía por parte de los investigadores los objetivos de la investigación, siempre con una prioridad a la libre construcción del discurso por parte del entrevistado y mínimas reconducciones por parte del entrevistador para favorecer la aparición de información relevante.

2) Más abierta aún ha sido la observación de campo, pues se ha llevado a cabo primando la inmersión natural en las aulas de audición y lenguaje implicadas en el desarrollo de la investigación, con declarada, expresa y compartida con los maestros la facción de observación, trasladando después de forma minuciosa al cuaderno de campo la información contingente con relación a los objetivos.

3) El análisis de los documentos de los maestros de audición y lenguaje y de los equipos de orientación educativa participantes, tanto de planificación como de evaluación y memoria, ha sido realizado para la obtención de información añadida, así como para la triangulación metodológica de la información aportada en entrevistas y observación.

\section{Procedimiento}

El procedimiento se ha sustentado en una inmersión múltiple en el campo, con nueve focos, a los que se llega con negociaciones individuales para cada foco. Durante la inmersión se han alternado las entrevistas en profundidad con diálogos espontáneos con distintos actores que se han ido registrando en el cuaderno de campo, a la vez que se acopiaban documentos para su posterior análisis.

Los nueve focos, constituidos por maestros itinerantes de audición y lenguaje, ubicados en siete equipos de orientación educativa de la provincia de Almería (España), han sido abordados como contextos de inmersión durante periodos de seis semanas con un día, sesión escolar completa, de estancia semanal con cada de ellos. Se descartaron los meses de septiembre y octubre para que la inmersión coincidiese con un periodo de actuación de 
los AL ya exento de las incertidumbres organizativas de inicio de curso frecuentemente presentes en la atención itinerante. Este planteamiento generó cuatro contextos de inmersión: dos contextos constituidos por los dos EOE que tenían dos AL en su plantilla, y otros dos contextos de dos y tres AL cada uno, agrupándolos por criterios de proximidad geográfica para facilitar la operatoria del propio proceso de inmersión. Eso llevó a un período de inmersión total de 24 semanas efectivas, lo que permitió terminar antes de junio, y así eludir un tiempo con un nivel de incidencias organizativas y curriculares generalmente más alto.

En términos de sesiones eso ha conducido a tener con varios de ellos las seis sesiones de contacto, otro grupo con alguna incidencia cinco y en un caso cuatro. En todos los casos la aproximación al AL, se articuló de forma personal y directa, así como simultánea a la gestión ante el equipo directivo, dentro del marco de colaboración que el grupo de investigación de pertenencia mantiene para diversas actividades con la Delegación de Educación de la provincia de Almería. Tras el período de inmersión se procedió al tratamiento de la información, con alguna visita adicional posterior para confrontar datos.

\section{Resultados}

El dato inicial que llama la atención acerca de la experiencia previa de estos profesionales es la diversidad de puestos que han venido ocupando con anterioridad, si bien, en su conjunto, todos son relativos al ámbito docente.

Así, entre el colectivo objeto de esta investigación, predominan los profesionales que han accedido después de haber impartido docencia preferente como maestros en Educación Primaria o Educación Infantil (E.AL2,3,5,8) y aquellos que han experimentado la función de maestro de Pedagogía Terapéutica o incluso la de Educación Especial-en Secundaria(E.AL1,4,7).

También existen casos de maestros de audición y lenguaje itinerantes que han ejercido con anterioridad periodos como AL de centro con dedicación plena (Andalucía) (E.AL7) o compartiendo periodos de prevención de dificultades de lenguaje oral con otras tareas docentes de carácter compensatorio (puesto desempeñado en Extremadura) (E.AL2), así como el caso de un profesional que reconoce haber trabajado como Logopeda en un gabinete a nivel privado (E.AL4).

En cuanto al periodo de experiencia en la función actual, la mayor parte de los encuestados mantiene una experiencia dilatada como AL itinerante ( $E . A L 1,2,3,4,5,7,8,9$ ) que les hace sentirse seguros en su función, sabedores de las limitaciones u obstáculos que presenta y un tanto conformistas con su perfil.

\footnotetext{
${ }^{1}$ Las citas textuales de datos empíricos de carácter cualitativo, se realiza en la parte de resultados utilizando un par de claves entre paréntesis, por ejemplo: $(E, A L 7)$. El primer componente del par es una letra mayúscula que representa la instrumento que da origen al dato (E, CC ó $A D)$, y el segundo componente del par representa al informante como los tipifica la Tabla 2, con la particularidad de que si se trata de un AL se le añade el número del 1 al 9 que los diferencia entre sí (Tabla 1).
} 


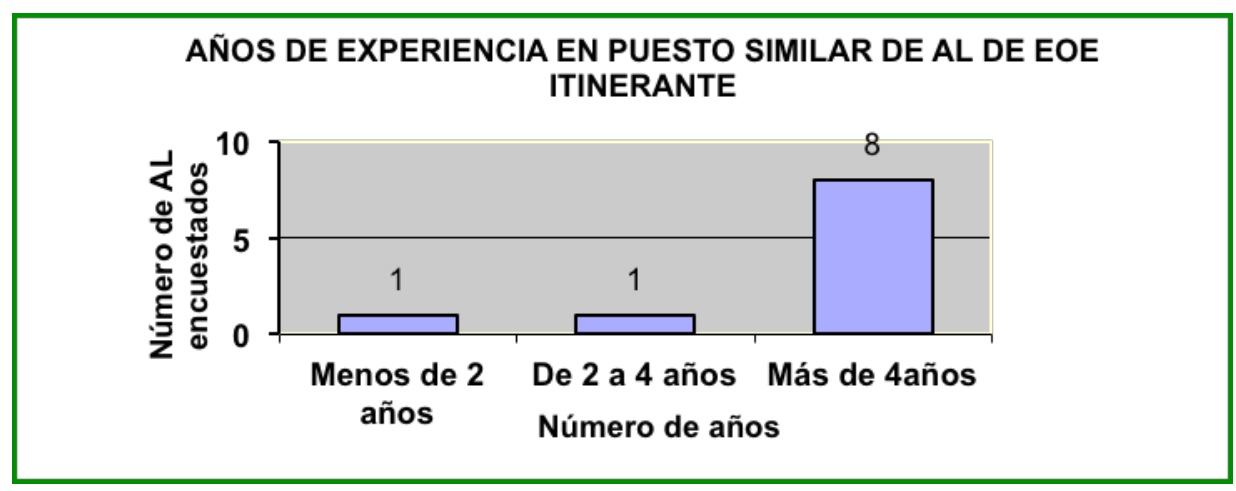

Gráfico 1: Años de experiencia en puesto similar de AL de EOE itinerante

Con relación a su sistema de acceso al puesto actual existe igualmente variedad, predominando los profesionales que, una vez han adquirido su plaza definitiva en Infantil o Primaria, se presentaron a unos concursos de Puestos de Carácter Singular que anualmente han venido convocando las Delegaciones Provinciales.

Una vez dichos profesionales han accedido a estas plazas, suelen ser renovados en su puesto año tras año en régimen de comisión de servicios, si bien ha llegado a darse la circunstancia de quedar plazas sin cubrir y empezar a utilizar la Administración el colectivo de la bolsa de interinos una vez comenzado el curso académico, los cuales se ven en la necesidad de acomodarse rápidamente al perfil que se hubiera venido desarrollando hasta entonces.

De todas formas, la inseguridad de permanecer ocupando dicho puesto puede ser la tónica que se respira en estos profesionales:

"Este puesto no se ocupa con definitivos. Ahora mismo yo estoy, he pedido la renovación como una comisión de servicios..." (E.AL2).

"Y ahora yo lo que estoy pidiendo es la renovación, que este año también la he pedido para el curso que viene. No sé si me la concederán o no me la concederán" (E.AL5).

"No saben si seguirán el año siguiente y eso se nota en la tranquilidad de su trabajo..." (E.Ma).

Con relación a la evolución de las plazas, nos indican que no han aumentado en número desde su implantación allá por los años noventa (CC.Or), existiendo demandas de incremento de plantilla reiteradas que nunca han sido atendidas (CC.AL4,9).

Los motivos de haber escogido dicho puesto también son variados, existiendo manifestaciones tan diversas como las ganas de hacer algo distinto como docente (CC.AL4), la independencia que propicia (CC.AL8), el gusto por la logopedia (CC.AL9), o simplemente la necesidad de trabajar o acercarse a su domicilio desempeñando cualquier función para la que estuvieran habilitados:

"Nosotros cogemos toda la zona hasta Bayárcal pero no me importaba, con tal de estar por aquí cerca. Prefería coger esto antes de arriesgarme a que me mandasen a... No sé dónde." (E.AL1)

Por consiguiente se hace complejo definir la figura profesional del AL itinerante sobre la base de una experiencia profesional homogénea y unos intereses que unifiquen su perfil, reconociendo la existencia de una diversidad de perspectivas profesionales que constituirán 
un matiz diferenciador decisivo para las actitudes que desarrollarán en la construcción del currículo en la acción (Gimeno Sacristán, 1998).

Después de todo lo comentado podemos apuntar que una panorámica tan diversa en relación con la experiencia profesional comentada resultaría enriquecedora si existiera un tronco común en la vertiente formativa inicial o a lo largo del desarrollo profesional que garantizase la definición de un perfil propio, en la línea de lo apuntado por Gimeno Sacristán y Pérez Gómez (1987) cuando afirman que las posiciones o perspectivas ante el conocimiento de los docentes se suelen correlacionar con las especialidades universitarias que han cursado.

Pero, como veremos en el apartado siguiente, dicho colectivo no cuenta con dicha caracterización común en su vertiente formativa.

La formación inicial de los profesionales objeto de este estudio es la de maestro, si bien la especialidad cursada, en el $80 \%$ de los casos, no es la propia del puesto que desempeñan (Audición y Lenguaje), sino otras como Educación Infantil, Educación Primaria o Educación Física (E.AL1,2,3,5,6,7,8,9). En estos casos, como requisito para acceso a tal función, han precisado realizar un curso de especialización de los organizados o reconocidos por la Administración Educativa para adquisición de nuevas habilitaciones en la década de los noventa (CC.AL9) o han superado la oposición (o alguna prueba en el caso de interinos) en esta especialidad.

"Yo era especialista en ciencias humanas. Pero aquí lo que te contaba era si estabas habilitada o si tenías la especialidad de audición y lenguaje. Yo tengo la especialidad de audición y lenguaje por oposición." (E.AL2)

De esta manera, el conseguir dicha habilitación administrativa se convertía en el requisito para poder optar a unos puestos prometedores que parecían abrir nuevos horizontes profesionales y de destino a los docentes de Primaria, siendo numerosos los cursos realizados en dicho sentido para conseguir con premura una plantilla habilitada con un perfil que, hasta la fecha, no ha llegado a desarrollarse.

"Porque no había logopedas y había que formar logopedas y la forma de hacerlo, de que salieran logopedas, era esa; hacer un Convenio entre Universidades y el Ministerio de Educación y Ciencia y entonces así se formarían... Y de ahí salieron pues muchos logopedas." (E.AL7)

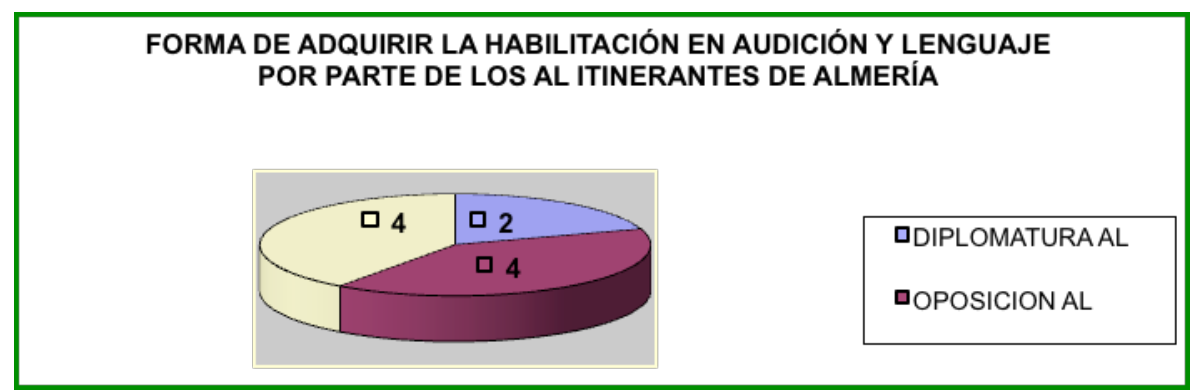

Gráfico 2: Forma de adquirir la habilitación en audición y lenguaje

Aquí nos encontramos con un factor importante que marcará la definición de este perfil profesional, pues si la formación inicial que han recibido la mayoría de los actuales AL durante su carrera no ha profundizado en la temática de su especialidad, dicho rol se vuelve más proclive a mantener las tendencias profesionales desarrolladas hasta la fecha, al recibir 
más importancia, por parte del profesional, los consejos de compañeros de trabajo o las recetas rápidas de aquellos que han pasado con anterioridad por el puesto.

"En cuanto a la formación inicial o universitaria, caber decir que, tanto yo como la mayoría de los que hoy en día nos dedicamos a la logopedia en Andalucía nos hemos formado en esta materia después de haber estudiado otra especialidad en la universidad, en Magisterio. Entonces, bueno, la mayoría nos hemos buscado luego la vida... La mayoría con cursos de especialización privados o habiendo aprobado alguna prueba de oposiciones. Luego también, he tenido la suerte de tener buenos maestros, de tener buenos compañeros que me han ayudado y a los que si les has consultado una duda o cualquier cosa siempre me han echado una mano." (E.AL9)

En este sentido, al ir profundizando a lo largo de las entrevistas con estos profesionales en la importancia que otorgan a la formación inicial que han recibido de cara al desempeño actual de su función, sorprende el hecho de que, a pesar de sentirse maestros (CC.AL4), consideran que los contenidos formativos de la carrera distan de los requerimientos que el puesto de maestro de AL itinerante precisa, lo cual va reflejando el carácter del perfil profesional que dichos profesionales han ido construyendo.

"En la carrera se ven las cosas muy superficialmente, se centran mucho en o que es la educación y todo pero nuestro trabajo yo creo que es muy especializado." (E.AL2)

Cabe reseñar el hecho de que sean los maestros que han cursado la especialidad de Audición y Lenguaje los que se quejan de la carga educativa y poco especializada que han recibido a lo largo de la carrera.

“CCuáles son los contenidos que más echo en falta en mi formación inicial? De tipo de la especialidad, tipo de trastornos, tipo de intervención con alumnos con las distintas alteraciones del lenguaje." (E.AL2)

Así como de unos periodos de prácticas en los que, mayoritariamente, asistían a cursos generalistas de Primaria o, en el mejor de los casos, observaban ocasionalmente las tareas que desempeñaba un profesional de AL de centro que en nada se parecían a las que se les demanda en el puesto actual.

"Mi formación inicial no es buena para esto. Yo cuando llegué desconocía lo que tenía que hacer y cómo hacerlo. (...) echo en falta de la formación de maestra AL más prácticas. En el primer y segundo periodo estuve en cursos de Primaria y sólo en tercero estuve con un maestro de AL pero es insuficiente. Yo estaba con la logopeda mirándola todo el rato, era un centro específico y no hice unas prácticas que me sirvieran para aprender yo a desempeñarme. Lo que hago ahora es distinto a todo eso que yo vi en mi formación." (E.AL8)

La razón de esto la podríamos encontrar en que, a raíz de los factores que venimos comentando (premura en la habilitación, formación inicial mayoritaria no específica, tendencia al conservadurismo en el rol) el nuevo perfil de maestro de AL itinerante no ha llegado a instaurarse con solidez, demandando dichos profesionales mayor carga curricular en contenidos de tipo clínico-terapéutico.

"Entonces yo creo que a mí no me formaron como especialista en logopedia." (E.AL2)

Esto también puede hacernos reflexionar sobre los obstáculos que las Universidades han venido encontrando a la hora de incidir en dicha formación inicial con carácter didáctico como maestro de $\mathrm{AL}$, requiriéndose una necesaria coordinación entre administraciones educativas (universitarias y no universitarias) a la hora de compaginar los ámbitos 
formativos (inicial/permanente) y los laborales, máxime en momentos de cambio o reforma.

Así, lo primero que destaca a lo largo de los cuestionarios y entrevistas realizadas en la presente investigación, es la opinión generalizada por parte de los maestros $A L$ acerca de la escasa o nula oferta de actividades formativas adecuadas para el puesto en cuestión (AL itinerante) e incluso para potenciar cualquier competencia profesional relacionada con la atención a la diversidad del alumnado (C.AL1,3,4,5,6,7,8,9).

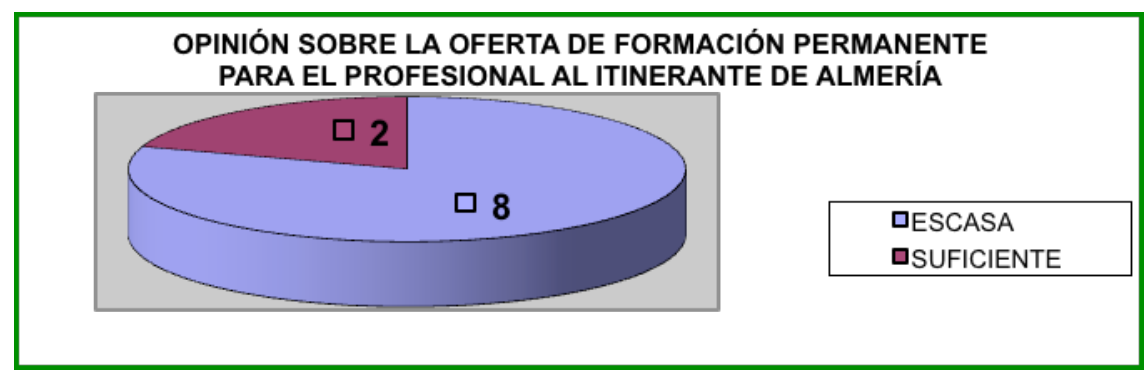

Gráfico 3: Opinión sobre la oferta de formación permanente

En un momento como el actual, en el cual la demanda generalizada de calidad por parte del sistema educativo y su relación manifiesta con la faceta formativa es una realidad palpable, los Centros de Profesorado (CEPs) centran su atención en las continuas demandas de actividades formativas que se vienen produciendo en aspectos novedosos como las TIC, el bilingüismo, la convivencia o la reciente implantación de los nuevos Reglamentos Orgánicos en los centros, temas que por su actualidad absorben la mayor parte de la oferta institucional. Actuaciones como las relativas al desarrollo de las competencias tutoriales, la promoción de estrategias de atención a la diversidad y concretamente la atención en el desarrollo de la capacidad lingüística-comunicativa mediante estrategias educativas suelen quedar un tanto relegadas sin llegar a profundizar en la variedad de facetas que abarcarían su campo de acción educativa (Plan de Formación del CEP de Almería 2010/2011).

A lo largo del periodo de investigación, el colectivo afectado ha manifestado en diversas ocasiones sentirse falto de atención por parte de la Administración Educativa (CC.AL3,6,9), debiendo recurrir, en el caso de los más emprendedores, a buscar fórmulas de autoformación, como puede ser el continuar los estudios universitarios realizando una licenciatura (CC.AL5) o el realizar las escasas actividades que se organizan en torno a esta temática a pesar de su dispersión geográfica (CC.AL3,9). Sin dejar de valorar ambas medidas, sí se aprecian comentarios acerca de la necesaria formación permanente específica para este rol docente, mediante actuaciones que se desarrollen en su lugar de trabajo y a lo largo de la jornada laboral, demandando planteamientos incidentes en la dinámica cotidiana de tal función mediante fórmulas que combinen el enriquecimiento teórico con la aplicación práctica (CC.AL7,8), que irían en consonancia con las nuevas propuestas formativas a nivel universitario que se pretenden instaurar en la actual época de construcción del Espacio Europeo de Educación Superior (formación en centros, team teaching, problem based learning, etc.)

En relación con esta temática, podemos apreciar cómo en los documentos de planificación general se echa en falta una propuesta amplia de actuaciones formativas para el AL a nivel de zona/provincia, tanto con el resto de profesionales itinerantes como con los compañeros docentes de los diversos centros educativos (AD.AL1,2,3,4), (AD.DD) demandándose por 
este colectivo un incremento de actividades formativas en centro de índole colaborativo con el conjunto del profesorado (AD.AL5,8), (AD, CO) (CC.AL2,8).

"Sería conveniente que existieran muchas más actividades de formación especializada no sólo para nosotros sino para el resto de tutores, para que colaboren con nuestra tarea." (E.AL.7)

"Deberían haber actividades de formación en esta temática para el conjunto de los tutores, ya que tendríamos que colaborar con los logopedas" (E.Tu).

Igualmente, podemos apreciar una demanda generalizada por parte de este colectivo en las Memorias Finales de Curso (AD.AL9) hacia una mayor atención de la Administración Educativa de cara a potenciar actividades formativas que faciliten el conjunto de funciones y responsabilidades diversas que desde dicha institución se le encomienda y que vienen desarrollando en amplias zonas de actuación (CC.Or).

Otro aspecto que se menciona por parte de los entrevistados es la superficialidad de las escasas actividades organizadas que distan de complementar la formación inicial recibida y se centran en temáticas actuales pero carentes de practicidad y rigor científico.

“Actividades de formación adecuadas para esta temática? No, y mucho menos de carácter científico. Yo pienso que estamos relegados, que tendríamos que tener más formación." (E.AL6)

"Existe poca oferta formativa para nosotros. Tendría que mejorar, la oferta debería ser mejor." (E.AL4)

"En la actualidad hay cursos donde la oferta de formación es más amplia y otros en los que es menor. Siempre es bueno que exista una oferta de formación lo más completa posible y además lo más actualizada posible y además que sea informativa y teórica pero sobre todo de aplicación práctica." (E.AL5)

A veces incluso se esgrime como motivo de inasistencia o queja frente a las convocatorias de estas actividades las dificultades que los profesionales itinerantes tienen para mantenerse informados de las mismas (CC.Or).

"Yo no he hecho ninguna porque no me han llegado de lo mío y que me interesen." (E.AL8)

"Hay pocos para los logopedas. Sobre todo algún tipo de trabajo con determinados aspectos de la educación especial, con audición y lenguaje, cursos y programas específicos. Yo creo que llega muy poca información para ellos." (E.PT)

Lo cual, sin dejar de ser real en cuanto a la escasa periodicidad de asistencia a reuniones de coordinación -que en algunos casos se limita a un par de horas semanales al utilizar parte de la sesión del viernes para ir a centros- (CC.AL8), no debería presentar más obstáculos que los propiamente organizativos de cualquier institución en cuanto a establecer unos periodos/sistemas de transmisión de información.

Otra modalidad formativa que se ha ido ensayando en los últimos años entre los AL itinerantes ha sido la existencia de grupos de trabajo de ámbito provincial, si bien se ha apreciado una decadencia en los últimos años (AD.AL1,2,3,4), (AD.DD) en razón de las frecuentes faltas de asistencia y discontinuidad en el trabajo desarrollado (CC.AL2,7). En este aspecto ha podido ser determinante la dispersión geográfica del colectivo y el número reducido de sus miembros.

"Luego, como somos tan poquitos y repartidos por tantos sitios pues tampoco tienes opción de organizar un grupo de trabajo." (E.AL1) 
Así como la inexistencia de un horario semanal establecido de dedicación a la formación, debiendo utilizar para ello periodos de coordinación en equipo o de atención a las familias y centros, los cuales en muchas ocasiones demandan su presencia y limitan la asistencia a dichas sesiones de trabajo en grupo (CC.AL2,6,9).

Es por eso que dichas reuniones han servido, en la mayoría de los casos, como puesta en común de problemáticas laborales o demandas administrativas, solucionario de dudas, lamento compartido y transmisión de novedades; aspectos éstos más de índole personal que profesional, que han provocado en unos casos la desidia y en otros la inasistencia a dichas sesiones.

"Lo de los grupos de trabajo a nivel nuestro está bien pero lo que pasa es que son reuniones que llegas cansado, llegas con muchos problemas. Entonces esas sesiones ¿sabes qué pasa? Se convierten un poco en consolarnos los unos a los otros, en contarnos nuestras penas y no se avanza lo que realmente se debería de avanzar o cumplir el objetivo para el cual íbamos." (E.AL3)

"También te vienes un poco liberado en el sentido de decir: ¡Bueno, a mi compañero le está pasando igual que a mí! Y eso sirve de consuelo. Algo de terapia es ¿no? Pero decir: ¡Pero si íbamos a esto no hemos hecho... ! Pero que en esas sesiones ha sido un poco de descarga de tensiones y..." (E.AL7)

De todas formas, según nos ha sido comentado por los partícipes es este estudio, ha habido cursos en los que dichas reuniones han funcionado y respondido a los intereses planteados en mayor o menor medida, habiendo elaborado modelos de informes evaluadores, iniciada la elaboración de programas de prevención de problemáticas en el desarrollo del lenguaje oral, etc. (CC.AL2,8). Pero dichas actuaciones se han caracterizado por su discontinuidad temporal y fragmentación geográfica, lo cual no ha dejado una sensación positiva en el conjunto del profesorado (CC.AL4,9).

No obstante, cabe señalar que en la actualidad las modalidades de formación continua están en permanente cambio, destacando la autoformación continua e innovación en el aula como una de las vías propicias para desarrollar las competencias generales que se indican en las propuestas de nuevos planes de estudio del alumnado universitario (concretamente en los alumnos de magisterio). Dicha modalidad autoformativa e innovadora -desarrollada fundamentalmente a través de grupos de trabajo o proyectos de formación en centros-, se considera de vital importancia para los requisitos profesionales del docente del siglo XXI (CRUE, 2006), señalándose como competencias profesionales a cuyo desarrollo colabora las siguientes:

- Reflexionar sobre las prácticas de aula para innovar y mejorar la labor docente.

- Asumir que el ejercicio de la función docente ha de ir perfeccionándose y adaptándose a los cambios científicos, pedagógicos y sociales a lo largo de la vida.

\section{Discusión y conclusiones}

El dato inicial que llama la atención acerca de la experiencia previa de estos profesionales es la diversidad de puestos que han venido ocupando con anterioridad, si bien, en su conjunto, todos son relativos al ámbito docente.

En cuanto al periodo de experiencia en la función actual, la mayor parte de los encuestados mantiene una experiencia dilatada como AL itinerante que les hace sentirse seguros en su función, sabedores de las limitaciones u obstáculos que presenta y un tanto conformistas con su perfil. 
La formación inicial que han recibido la mayoría de los actuales AL durante su carrera no ha profundizado en la temática de su especialidad, resultando escasos los bloques formativos dedicados a la vertiente didáctica de la faceta comunicacional. En cualquier caso, la mayoría de los profesionales AL de EOE de este estudio carecen de los requerimientos formativos de dicho puesto, tanto desde el punto de vista del modelo clínico como del curricular, siendo en su mayoría funcionarios/maestros -interinos en su mayor número- que han obtenido la habilitación administrativa para dicha función a través de la preparación de un temario de oposiciones (aprobar alguna prueba) o mediante un curso de especialización de unos meses (eso en el caso de no haber ingresado en bolsa con menos requisitos aún de los aquí mencionados), provocándose la rápida asunción del rol establecido por parte de los miembros que van ingresando en la realidad del mismo.

Destaca la opinión generalizada por parte de los maestros AL sobre la escasez de oferta formativa de carácter continuo para estos profesionales, así como la dispersión de la misma, y la dificultad de integrarse en actuaciones de formación en centros o de coordinarse con la multiplicidad de perfiles de los miembros del EOE, si bien nuestro profesional destaca en su interés y esfuerzo por aprovechar las oportunidades que se le brindan.

Con relación a su sistema de acceso al puesto actual existe igualmente variedad, predominando los profesionales que, una vez han adquirido su plaza definitiva en Infantil o Primaria, se presentaron a unos concursos de puestos de carácter singular que anualmente han venido convocando las delegaciones provinciales de educación.

Los motivos de haber escogido dicho puesto también son variados, existiendo manifestaciones tan diversas como las ganas de hacer algo distinto como docente, la independencia que propicia el gusto por la logopedia o simplemente la necesidad de trabajar o acercarse a su domicilio desempeñando cualquier función para la que estuvieran habilitados.

\section{Bibliografía}

Angulo Rasco, F. (1999). Entrenamiento y coaching: los peligros de una vía revitalizada. En F. Angulo Rasco, J. Barquín Ruiz y A.I. Pérez Gómez (Eds.), Desarrollo profesional del docente. Política, investigación y práctica (pp.467-505). Madrid: Akal.

Clarke, D. y Hollingsworth, H. (2002). Elaborating a model or teacher professional growth. Teaching and Teacher Education, 18 (1), 947-967.

Consejería de Educación de la Junta de Andalucía (2010). Plan de Formación del CEP de Almería 10/11. <http://www.cepalmeria.org> [Consulta: 1 oct. 2010]

CRUE (2006). Ficha Técnica de Propuesta de Título Universitario de Grado en Magisterio de Educación Primaria, según RD 55/2005, de 21 de enero. CRUENOTICIAS, $N^{\circ} 2$-Enero2006.<http://www.crue.org/BOLETINES/BOLETIN_N2/Boletin2.htm\#polituni> [Consulta: 8 Mayo, 2006]

Escudero Muñoz, J.M.(1993). Formación en centros e innovación educativa. Cuadernos de Pedagogía, 220, 11-17.

Fernández Sierra, J. y Barquín Ruiz, J. (1998).Opinión del profesorado andaluz sobre la formación permanente: estudio de dos generaciones de docentes, Revista de Educación, 317, 281-300.

Fullan, M. (1993). Why Teacher Must Become Change Agents. Educational Leadership, marzo, $12-17$. 
Gimeno Sacristán, J. (1998). El curriculum: una reflexión sobre la práctica. Madrid: Morata.

Gimeno Sacristán, J. y Pérez Gómez, A.I. (1987). El pensamiento psicopedagógico de los profesores (Informe de investigación). Madrid: CIDE.

Gimeno Sacristán, J., Pérez Gómez, A., Martínez Rodríguez, J., Torres Santomé, J., Angulo Rasco, F. y Álvarez Méndez, J. M. (2009). Educar por competencias, ¿qué hay de nuevo? Madrid: Morata.

Goetz, J.P. y LeCompte, M.D. (1988). Etnografía y diseño cualitativo en investigación educativa. Madrid: Morata.

Habermas, J. (1987). Teoría de la acción comunicativa. Taurus: Madrid.

Imbernóm Muñoz, F. (2005). ¿Qué formación permanente?. Cuadernos de Pedagogía, 348, Julio-Agosto, 70-73.

Luque de la Rosa, A. (2008). El modelo de intervención itinerante en el maestro de audición y lenguaje. Revista Electrónica Interuniversitaria de Formación del Profesorado REIFOP, 11 (1), 45-51.

Nuño Pérez, J. (1991). La formación de los futuros maestros: especialidad Audición y Lenguaje. Revista Interuniversitaria de Formación del Profesorado RIFOP, 12, 167-179.

Pérez Gómez, Á.(2002). La función y formación del profesor/a en la enseñanza para la comprensión. En J. Gimeno Sacristán y Á.l. Pérez Gómez (Eds.), Comprender y transformar la enseñanza (10 edición) (pp.398-429). Madrid: Morata.

Pérez Gómez, Á.l. (1995). Autonomía profesional del docente y control democrático de la práctica educativa. En F. Alfieri y otros (Coords.), Volver a pensar la educación (Vol. II) Prácticas y discursos educativos (Congreso Internacional de didáctica) (pp.339353). Madrid: Morata.

Real Decreto 1594/2011, de 4 de noviembre, por el que se establecen las especialidades docentes del Cuerpo de Maestros que desempeñen sus funciones en las etapas de Educación Infantil y de Educación Primaria reguladas en la Ley Orgánica 2/2006, de 3 de mayo, de Educación (BOE 9-11-2011).

Rodríguez Gómez, G., Gil Flores, J. y García Jiménez, E. (1996). Metodología de la Investigación Cualitativa. Málaga: Ediciones Aljibe.

Schön, D. A. (1983). The reflective practitioner. Londres: Temple Smith.

Stake, R.E. (1994). Case Studies. En N.K. Denzin e Y.S. Lincoln (Eds.), Handbook of qualitative research (pp.236-247). Thousand Oaks, CA: Sage Publications.

Stake, R.E. (1995). The Art of Case Study Research. Thousand Oaks, CA: Sage Publications.

Taylor, S.J. y Bogdan, R. (1986). Introducción a los métodos cualitativos de investigación. Barcelona: Paidós.

Tikunoff, W.Y. (1979). Context variables of a teaching-learning events. En D. Benet y D. McNamara (Eds.), Focus on teaching readings in the observations and conceptualization of teaching (pp.46-59). Nueva York: Longman.

Woods, P. (1989). La escuela por dentro: la etnografía en la investigación educativa. Barcelona: Paidós. 


\section{Autores}

Antonio Luque de la Rosa

Nacido el 4 de enero de 1967 en Priego de Córdoba (Córdoba). Maestro por la especialidades de matemáticas, ciencias de la naturaleza y audición y lenguaje (Primer Premio Nacional de Terminación de Estudios), Licenciado en Filosofía y Ciencias de la Educación por la UNED y Doctor en Innovación Educativa por la Universidad de Almería.

Tras una dilatada y variada carrera docente, actualmente es profesor universitario en el Departamento de Educación de la Universidad de Almería, desempeñando el cargo de Director del Secretariado de Orientación Educativa y Vocacional en esta universidad

\section{José Juan Carrión Martínez}

Natural de Lucainena de las Torres (1957). Maestro especialista en Ciencias, Educación Especial y Audición y Lenguaje (Universidad de Granada), Licenciado en Ciencias de la Educación Universidad Autónoma de Barcelona y UNED), Doctor en Psicopedagogía (Universidad de Almería)

Actualmente es Profesor Titular de Universidad en el Departamento de Educación de la Universidad de Almería con docencia en grado, máster oficial y doctorado: materias de educación especial, asesoramiento en educación y orientación (1995-hasta la fecha) y ostenta el cargo de Vicerrector de Profesorado y Ordenación Académica desde septiembre 2008 